\title{
Soluble Human Leukocyte Antigen-G Expression in Pregnancy Success and Early Pregnancy Loss in Korle-Bu Teaching Hospital
}

\author{
Abdul-Rahman Mubarak ${ }^{1}$, Irene Sitsofe Blebu ${ }^{1}$, Kareem Mumuni' ${ }^{2}$, Yao Tettey ${ }^{1}$, \\ Richard Kwasi Gyasi' ${ }^{1}$ Andrew Anthony Adjei', Michael Ofori ${ }^{3}$ \\ ${ }^{1}$ Department of Pathology, School of Biomedical and Allied Health Sciences, University of Ghana, Accra, Ghana \\ ${ }^{2}$ Department of Obstetrics and Gynaecology, School of Medicine and Dentistry, University of Ghana, Accra, \\ Ghana \\ ${ }^{3}$ Department of Immunology, Noguchi Memorial Institute of Medical Research, Accra, Ghana \\ Email: "presmubarak@gmail.com
}

Received 11 November 2015; accepted 28 February 2016; published 2 March 2016

Copyright (C) 2016 by authors and Scientific Research Publishing Inc.

This work is licensed under the Creative Commons Attribution International License (CC BY).

http://creativecommons.org/licenses/by/4.0/

c) (i) Open Access

\begin{abstract}
Introduction: Human leukocyte antigen G (HLA-G) is a non-classical major histocompatibility complex (MHC) class Ib antigen characterized by a limited polymorphism. The expression of HLA-G at immune privileged sites and its ability to inhibit the effectors functions of immune cells has set HLA-G as a molecule of immune tolerance. This expression pattern is unique among HLA genes and suggests that HLA-G may be involved in interactions that are critical in establishing and/or maintaining pregnancy. Methods: Soluble HLA-G (sHLA-G) levels were measured using a BioVendor sHLA-G ELISA kit following the manufacturer's protocol. The study participants include women undergoing spontaneous abortion, non-pregnant women, males and an archive sample of women who had normal vaginal deliveries without any complications and any history of malaria infection from gestation to delivery. Results: Soluble HLA-G levels were higher among women undergoing spontaneous abortion as compared to women who had normal vaginal delivery and non-pregnant women. Soluble HLA-G levels were also higher in second trimester as compared to first trimester in both women who had spontaneous abortions and women who had normal delivery. Conclusion: Although sHLA-G levels were higher among women undergoing spontaneous abortion as compared to non-pregnant women and women who had normal delivery, this may be playing a role in the maintenance of maternal immune tolerance to fetal antigen, since plasma sHLA-G levels increased with increasing trimester in both women who had normal delivery and women undergoing spontaneous abortion.
\end{abstract}

${ }^{*}$ Corresponding author.

How to cite this paper: Mubarak, A.-R., Blebu, I.S., Mumuni, K., Tettey, Y., Gyasi, R.K., Adjei, A.A. and Ofori, M. (2016) Soluble Human Leukocyte Antigen-G Expression in Pregnancy Success and Early Pregnancy Loss in Korle-Bu Teaching Hospital. Open Journal of Immunology, 6, 1-6. http://dx.doi.org/10.4236/oji.2016.61001 


\section{Keywords}

\section{Soluble Human Leukocyte Antigen G (sHLA-G), Spontaneous Abortion, Maternal Immune Tolerance Implantation}

\section{Introduction}

The term HLA refers to the Human Leucocyte Antigen System, which is controlled by genes on the short arm of chromosome six. The HLA loci are part of the genetic region known as the Major Histocompatibility Complex (MHC) [1]. The MHC, the most gene-dense region of the human genome, contains at least 128 functional genes, more than $20 \%$ of which have functions in immunity. It is also the region with the most disease associations. The MHC genes are traditionally divided into three classes. Genes located in the region of MHC class I and II encode the antigen-presenting MHC molecules while class III genes encode complement hormones and other proteins [2]. The peptide antigen-presenting MHC molecules are known as classical MHC molecules. Structurally related molecules of class I and II that do not function in the presentation of peptide antigens are known as non-classical MHC molecules.

Human leukocyte antigen G (HLA-G) is a non-classical major histocompatibility complex (MHC) class Ib antigen characterized by a limited polymorphism. In non-pathological situations, HLA-G expression is restricted to the fetal-maternal interface of the extravillous cytotrophoblasts, placental chorionic endothelium, thymic epithelial cells, and erythropoietic lineage cells from the bone marrow, as well as other immune-privileged tissues such as the cornea, nail matrix, and autologous tissues such as the pancreas [3]. Functionally, HLA-G inhibits the cytolytic function of uterine and peripheral blood NK cells, the antigen-specific cytolytic function of cytotoxic $\mathrm{T}$ lymphocytes, the alloproliferative response of CD4 $\mathrm{T}$ cells, the proliferation of $\mathrm{T}$ cells and peripheral blood NK cells and the maturation and function of dendritic cells [4] [5]. The expression of HLA-G at immune privileged sites and its ability to inhibit the effectors functions of T-lymphocytes, natural killer cells (NK) and antigen-presenting cells (APC) has set HLA-G as a molecule of immune tolerance. Soluble HLA-G was detected in pregnant women from gestation to term, umbilical cord blood samples, amniotic fluid, and is found in the supernatant culture media from in vitro cultured embryos [6]-[8]. Functionally high soluble HLA-G expression is associated with successful organ transplantation, maintenance of maternal immune tolerance, successful in vitro fertilization therapy as a marker for embryo quality [9]. Decreased plasma level of soluble HLA-G in early gestation is associated with later development of preeclampsia and intrauterine growth retardation [10]. This expression pattern suggests that HLA-G may be involved in interactions that are critical in establishing and/or maintaining pregnancy. Therefore the aim of this study is to determine the role of sHLA-G expression in pregnancy success and early pregnancy loss in Korle-Bu Teaching Hospital (KBTH).

\section{Methods}

\subsection{Study Site and Target Population}

The study participants include women undergoing spontaneous abortion recruited from the Gynecology Unit KBTH and non-pregnant women and healthy men recruited from the staff of College of Health Sciences. An archive sample of women who had normal vaginal deliveries without any complications and any history of malaria infection from gestation to delivery was obtained from Noguchi Memorial Institute for Medical Research Ghana. KBTH situated in the nation's capital, Accra, Ghana, is the leading tertiary hospital and the major referral center in the country. It also serves as the teaching hospital of the University of Ghana College of Health Sciences, in Accra. Participants with history of immunodeficiencies, malignancies, and immunosuppressive and immune stimulant therapies were excluded from the study.

\subsection{Sample Collection and Analysis}

Five milliliters $(5 \mathrm{ml})$ of venous blood was obtained from each consented participant using butterfly needles ( 0.8 $\times 19 \mathrm{~mm} \times 178 \mathrm{~mm}, \mathrm{BD}$, USA) into heparinized vacutainers (BD, USA) tubes. Blood samples were transported 
to the laboratory in ice chests within one hour of sample collection. Plasma was separated from heparinized blood samples by centrifugation from whole blood at $2000 \mathrm{rpm}$ for 10 minutes. After centrifugation the plasma was collected and placed in cryotubes and stored at $-80^{\circ} \mathrm{C}$ until ready to use.

\subsection{Soluble HLA-G Measurement}

The sHLA-G levels were measured using a BioVendor sHLAG ELISA kit following the manufacturer's protocol. The frozen plasma samples were allowed to thaw at room temperature $\left(25^{\circ} \mathrm{C}\right)$ and diluted ten times with dilution buffer and vortex. $100 \mu \mathrm{l}$ of each of the diluted plasma samples were added to microplate wells pre-coated with monoclonal anti sHLA-G antibody and incubated at $2^{\circ} \mathrm{C}-8^{\circ} \mathrm{C}$ for 18 hours without shaking. After the incubation, the plate was washed manually for five times $(5 \times)$ with $0.35 \mathrm{ml}$ of wash solution per well. The microtiter plate was then bloated on paper towel store move excess wash solution and unbound analytes. After the washing, $100 \mu \mathrm{l}$ of Conjugate Solution containing monoclonal anti-human $\beta_{2}$ macroglobulin antibody labeled with horse radish peroxidase was added. The microtiter plate was then incubated on anorbital plate shaker with shaking at $300 \mathrm{rpm}$ for 1 hour at room temperature $\left(25^{\circ} \mathrm{C}\right)$. Following another washing step, to each well, $100 \mu \mathrm{l}$ of Substrate Solution containing tetramethylbenzidine was added and incubated for 25 minute at room temperature $\left(25^{\circ} \mathrm{C}\right)$ without shaking. After incubation, the color development was stopped by addition of $100 \mu$ of Stop Solution. The absorbance of each well was determined using microplate reader set to $450 \mathrm{~nm}$ with a reference wavelength of $630 \mathrm{~nm}$ within 5 minutes following addition of stop solution. Spectrophotometric readings of the assays were done both at $650 \mathrm{~nm}$ and $450 \mathrm{~nm}$, and the readings were subtracted to obtain the various absorbance.

\section{Statistical Analysis}

Statistical analysis was performed using Sigma-Stat Version 3.5. The differences of sHLA-G levels between two groups were tested using the nonparametric Mann-Whitney $U$ test. The differences among three or more groups were tested using the nonparametric Kruskal-Wallis test. $P$-value $<0.05$ was considered statistical significant.

\section{Results}

A total of eighty (80) study participant samples were analyzed. This includes thirty-two (32 (40\%)) women undergoing spontaneous abortion (SA) and twenty eight (28 (35\%)) archive samples of women who had normal vaginal deliveries without any complications and any history of malaria infection from gestation to delivery, ten (10 (12.5\%)) non-pregnant women and ten (10 (12.5\%) normal healthy men (NM) as shown in Table 1.

Soluble HLA-G levels were higher in women undergoing spontaneous abortion (66.5 $\mathrm{U} / \mathrm{ml})$ as compared to women who had normal vaginal delivery $(49.35 \mathrm{U} / \mathrm{ml})$ and non-pregnant women $(58.28 \mathrm{U} / \mathrm{ml})$. Generally higher Soluble HLA-G Level was observed in males $(79.11 \mathrm{U} / \mathrm{ml})$ as compared to all the study groups as shown in Figure 1.

Moreover, sHLA-G levels were higher in women who had spontaneous abortions as compared to women who had normal delivery in both first and second trimester, although it was not statistically significant (first trimester: spontaneous abortion $=66.53 \mathrm{U} / \mathrm{ml}$, women who had normal delivery $=41.94 \mathrm{U} / \mathrm{ml}$, second trimester: spontaneous abortions $=98.65 \mathrm{U} / \mathrm{ml}$, women who had normal delivery $=69.01 \mathrm{U} / \mathrm{ml}, P=0.09$ ). Although not statistically significant, sHLA-G levels were higher in second trimester as compared to first trimester in both women who had spontaneous abortions and women who had normal delivery as shown in Figure 2.

Table 1. Demographic characteristics of study participants.

\begin{tabular}{ccc}
\hline Study group & N & Age (M \pm SD) \\
\hline Spontaneous abortion (SA) & $32(40 \%)$ & $31.7 \pm 3.2$ \\
Normal delivery women (NDW) & $28(35 \%)$ & $24.6 \pm 2.4$ \\
Non-pregnant women (NPW) & $10(12.5 \%)$ & $22.6 \pm 3.7$ \\
Health adult men (NM) & $10(12.5 \%)$ & $25.6 \pm 5.2$ \\
\hline
\end{tabular}




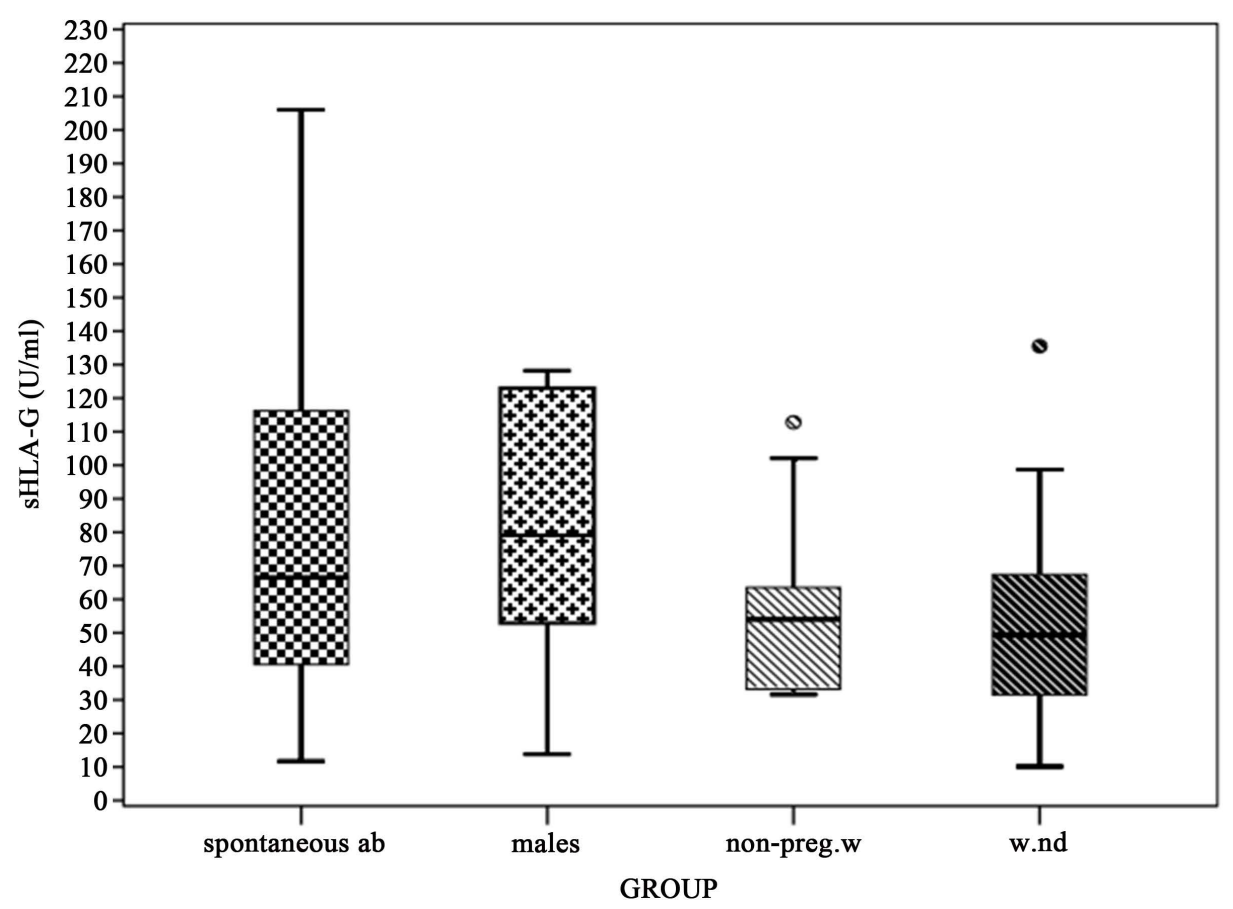

Figure 1. sHLA-G levels in spontaneous abortion patients (spontaneous ab), non-pregnant women (non-preg.w), women who had normal delivery (w.nd) and healthy adult males.

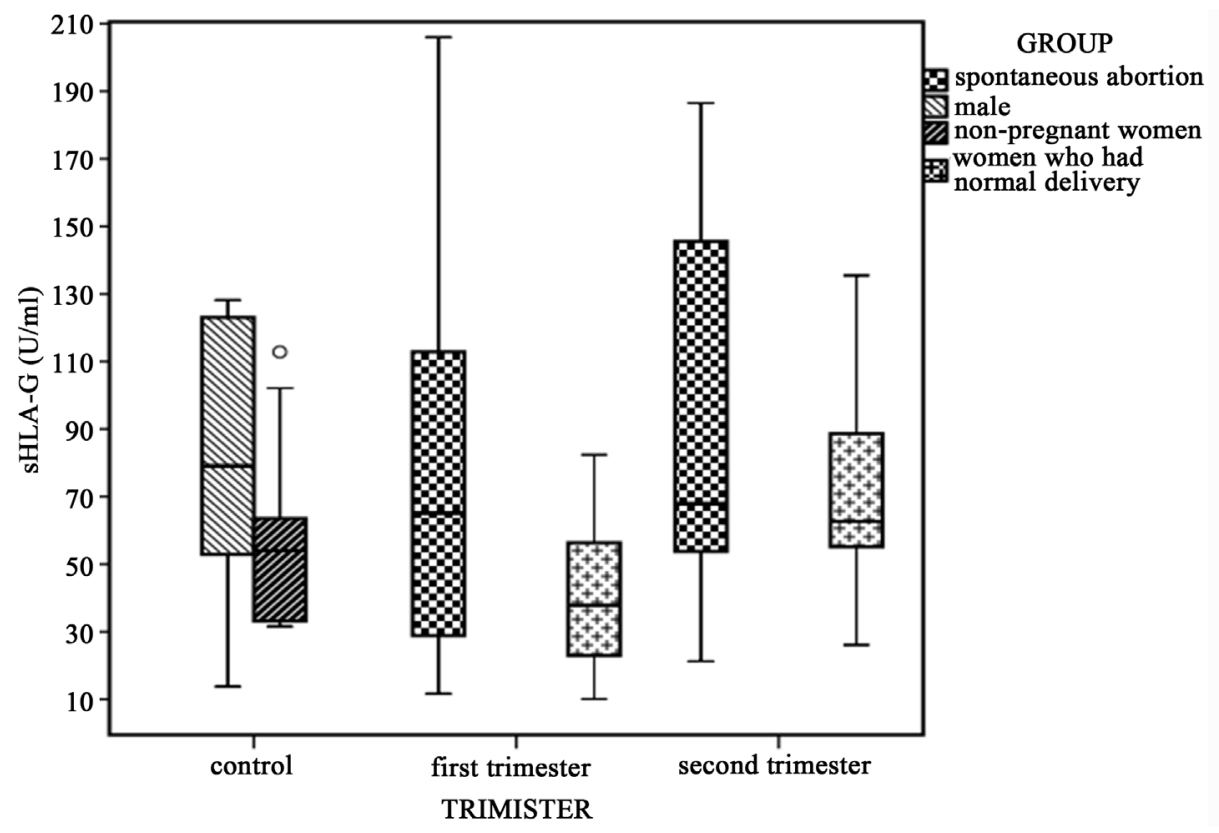

Figure 2. sHLA-G levels in controls (healthy adult male and non-pregnant women), women who had spontaneous abortion and women who had normal delivery in first and second trimester.

\section{Discussion}

Fetal survival requires mechanisms that allow immunologic tolerance of the fetal allograft by the mother and at the same time maintaining immune competence against invading pathogens. It is becoming clear that human reproduction is a dynamic interplay between immunological and immunogenetic factors during pregnancy. The alteration of these factors may lead to reproductive failure, although this remains a relatively unexplored area of 
research. Spontaneous abortion is one of the least understood pathological processes, in spite of being one of the most common pregnancy complications.

Human leukocyte antigen was found to play a critical role in pregnancy which includes gamete development, embryo cleavage, blastocyst and trophoblast formation, fetal development and survival [11]. Most studies have shown how HLA-G is involved in creating tolerance. For example, sHLA-G was found to play a role in suppressing NK cell activity, regulating cytokines production, inducing apoptosis in $\mathrm{T}$ cells and inhibiting the activation of immune cells [12]. sHLA-G was also found to play a role in trophoblast invasion and maintenance of successful implantation. Although some studies have shown a positive correlation between high HLA-G levels and successful implantation, in this study, high plasma HLA-G levels were found to be associated with poor implantation. This is evidence in the fact that high plasma HLA-G levels were observed in males, non-pregnant females and females undergoing spontaneous abortion as compared to women who had normal delivery. This observation is also in agreement with a study by Hviid and colleagues in 2006 who have shown that sHLA-G positive pre implantation embryos fail to implant in IVF treatment and levels of HLA-G and interleukin-10 (IL-10) cytokine believed to be of central importance in pregnancy, are not linked [11]. Furthermore McCormick et al., (2009) reported that sHLA-G actually inhibit trophoblast invasion of the maternal deciduas [13]. Dahl and colleagues in 2014 also reported that HLA-G may inhibit trophoblast invasion in concentrationdependent manners [14]. It could be that sHLA-G may not play a role in implantation, but rather in the maintenance of maternal immune tolerance to fetal antigen after implantation, because plasma sHLA-G levels increased with increasing trimester in both women who had normal delivery and women undergoing spontaneous abortion in this study. The third trimester plasma sHLA-G level of women who had normal delivery was found to be high as compared to their second trimester plasma sHLA-G level. Similar trends were observed in women undergoing spontaneous abortion.

\section{Limitation}

The use of archival sample of women who had normal vaginal deliveries might have affected the sHLA-G measured inthis group of study participant.

\section{Conclusion}

Evidence for the role of HLA-G in pregnancy still remains unclear. Many controversies surround HLA-G functions in pregnancy and its complications. It could be that sHLA-G may not play a role in implantation, but rather in the maintenance of maternal immune tolerance to fetal antigen after implantation, because plasma sHLA-G levels increased with increasing trimester in both women who had normal delivery and women undergoing spontaneous abortion. Also, sHLA-G is detectable in males and females under normal condition, with males showing the highest plasma levels of sHLA-G.

\section{Acknowledgements}

Hosu-Porbley, Sanela Setutsi Adzotor Hosu-Porbley.

\section{References}

[1] Shankarkumar, U. (2004) The Human Leukocyte Antigen (HLA) System. International Journal of Human Genetics, 4, 91-103.

[2] William, E. (2003) Fundamental Immunology. 5th Edition, Lippincott Williams \& Wilkins Publishers, Bethesda, Chapter 19.

[3] Cai, M., Han, H., Bei, J., Liu, C., Lei, J., Cui, Q., Feng, Q., Wang, H., Zhang, J., Liang, Y., Chen, L., Kang, T. and Shao, J. (2012) Expression of Human Leukocyte Antigen G Is Associated with Prognosis in Nasopharyngeal Carcinoma. International Journal of Biological Sciences, 8, 891-900. http://dx.doi.org/10.7150/ijbs.4383

[4] Carosella, E.D., Favier, B., Rouas-Freiss, N., Moreau, P. and LeMaoult, J. (2012) Beyond the Increasing Complexity of the Immunomodulatory HLA-G Molecule. Blood, 111, 4862-4870. http://dx.doi.org/10.1182/blood-2007-12-127662

[5] Laird, S.M., Tuckerman, E.M., Cork, B.A., Linjawi, S., Blakemore, A.I.F. and Li, T.C. (2003) A Review of Immune Cells and Molecules in Women with Recurrent Miscarriage. Human Reproduction Update, 9, 163-174.

http://dx.doi.org/10.1093/humupd/dmg013 
[6] Noci, I., Fuzzi, B., Rizzo, R., Melchiorri, L., Criscuoli, L., Dabizzi, S., Biagiotti, R., Pellegrini, S., Menicucci, A. and Baricordi, O.R. (2005) Embryonic Soluble HLA-G as a Marker of Developmental Potential in Embryos. Human Reproduction, 20, 138-146. http://dx.doi.org/10.1093/humrep/deh572

[7] Čerkienè, Ž., Eidukaitè, A. and Usonienè, A. (2006) Soluble Human Leukocyte Antigen G in Early Human Embryo Cultures after Assisted Reproduction Procedures. Acta Medica Lituanica, 13, 226-231.

[8] Dahl, M., Klitkou, L., Christiansen, O.B., Djurisic, S., Piosik, Z.M., Skovbo, P., Møller, A.M., Steffensen, R. and Hviid, T.V.F. (2015) Human Leukocyte Antigen (HLA)-G during Pregnancy Part I: Associations between Maternal and Fetal HLA-G Genotypes and Soluble HLA-G. Human Immunology, 76, 260-271. http://dx.doi.org/10.1016/j.humimm.2015.01.015

[9] Hunt, J.S., Langat, D.K., McIntire, R.H. and Morales, P.J. (2006) The Role of HLA-G in Human Pregnancy. Reproductive Biology and Endocrinology, 4, S10. http://dx.doi.org/10.1186/1477-7827-4-S1-S10

[10] Steinborn, A., Varkonyi, T., Scharf, A., Bahlmann, F., Klee, A. and Sohn, C. (2007) Early Detection of Decreased Soluble HLA-G Levels in the Maternal Circulation Predicts the Occurrence of Preeclampsia and Intrauterine Growth Retardation during Further Course of Pregnancy. American Journal of Reproductive Immunology, 57, 277-286. http://dx.doi.org/10.1111/j.1600-0897.2007.00475.x

[11] Hviid, T.V.F. (2006) HLA-G in Human Reproduction: Aspects of Genetics, Function and Pregnancy Complications. Human Reproduction Update, 12, 209-232. http://dx.doi.org/10.1093/humupd/dmi048

[12] Sargent, I.L. (2005) Does “Soluble” HLA-G Really Exist? Another Twist to the Tale. Molecular Human Reproduction, 11, 695-698. http://dx.doi.org/10.1093/molehr/gah196

[13] McCormick, J., Whitley, G.S.J., Le Bouteiller, P. and Cartwright, J.E. (2009) Soluble HLA-G Regulates Motility and Invasion of the Trophoblast-Derived Cell Line SGHPL-4. Human Reproduction, 24, 1339-1345. http://dx.doi.org/10.1093/humrep/dep026

[14] Dahl, M. and Hviid, T.V.F. (2012) Human Leucocyte Antigen Class Ib Molecules in Pregnancy Success and Early Pregnancy Loss. Human Reproduction Update, 18, 92-109. http://dx.doi.org/10.1093/humupd/dmr043 